\title{
A Pyroptosis-Associated Signature Plays a Role in Prognosis Prediction And Indicating Infiltration in Immune Microenvironment in Clear Cell Renal Cell Carcinoma
}

\section{Zhiyuan Li}

The First Affiliated Hospital of Harbin Medical University

\section{Zhinan Xia}

The First Affiliated Hospital of Harbin Medical University

\section{Yipeng Yu}

The First Affiliated Hospital of Harbin Medical University

\section{Licheng Cai}

The First Affiliated Hospital of Harbin Medical University

\section{Wengang Jian}

The First Affiliated Hospital of Harbin Medical University

\section{Tengda Wang}

The First Affiliated Hospital of Harbin Medical University

\section{Wei Xue}

The First Affiliated Hospital of Harbin Medical University

\section{Xingyuan Wang}

The First Affiliated Hospital of Harbin Medical University

\section{Bowen Wang}

The First Affiliated Hospital of Harbin Medical University

\section{Peng Zhang}

The First Affiliated Hospital of Harbin Medical University

\section{Wenhao Yao}

The First Affiliated Hospital of Harbin Medical University

\section{Cheng Zhang}

The First Affiliated Hospital of Harbin Medical University Chunyang Wang ( $\sim$ wangchunyang001@hotmail.com)

The First Affiliated Hospital of Harbin Medical University

\section{Research Article}


Keywords: clear cell renal cell carcinoma, pyroptosis, immune infiltration, prognosis, Multi-omics data, functional enrichment analysis, bioinformatics analysis

Posted Date: November 1st, 2021

DOl: https://doi.org/10.21203/rs.3.rs-995421/v1

License: (c) (1) This work is licensed under a Creative Commons Attribution 4.0 International License. Read Full License 


\section{Abstract}

Background: About 90\% renal malignancies are RCCs (renal cell carcinomas) and the main subtype in histology is ccRCC (clear cell RCC). In recent years, pyroptosis was considered as a kind of inflammationrelated programmed cell death, which played a part in the invasion, metastasis and proliferation of tumor cells, thereby influencing tumor prognosis. Nonetheless, the expression level of pyroptosis-associated genes in RCCs and its relationship with prognosis are still not clear.

Results: In our research, 44 regulators for pyroptosis were identified which were expressed differentially between normal kidney and ccRCC tissues. ccRCC cases were categorized into 2 subgroups with a significant difference between them in OS (overall survival) according to the DEGs (differentially expressed genes). The prognostic value of pyroptosis-associated genes was assessed as a signature based on the cohort of TCGA (The Cancer Genome Atlas). Following Cox regression with DEGs and LASSO (least absolute shrinkage and selection operator), a 6-gene signature had been set up and all the ccRCC cases in the cohort of TCGA were categorized into LR (low-risk) group or HR (high-risk) group (P < 0.001). In combination with clinical features, risk scores were considered as a predicting factor of OS in ccRCC cases. KEGG (Kyoto Encylopedia of Genes and Genomes) and GO (Gene ontology) analyses implied increased immunity and enrichment of genes related to immunity in the HR group.

Conclusions: Our findings indicated that the genes related to pyroptosis had an important role in tumor immunity, which were potentially used to predict the prognosis of ccRCCs.

\section{Introduction}

RCCs (renal cell carcinomas) are derived from renal tubular epithelial cells, accounting for approximately ninety percentage of all kinds of renal malignancies. The main histological type is clear cell RCC (ccRCC) [1]. Recently, RCCs incidence in most countries has elevated, with 400,000 new cases every year around world and over 175,000 deaths. Its mortality rate and incidence rank 3rd among urological malignancies worldwide[2, 3]. For regional renal cancer in early stage, radical nephrectomy is the first-line treatment, even though distant metastasis or tumor recurrence still remains unsolved after operation in over $20 \%$ of patients [4]. Meanwhile, RCCs are characterized by tolerance to chemotherapy and radiotherapy. Developing a specific prognostic strategy is of great importance to improve therapeutic effects.

Pyroptosis is a novel way of programmed cell death, which is recognized as inflammation related necrosis of cells [5], which is induced by a variety of stimuli, including heart attack, bacterial or virus infections, cancer and stroke [6]. Except for autophagy, apoptosis, and ferroptosis, this kind of cell death has attracted much attention recently.

The characteristics of pyroptosis is cell swelling, large bubbles moving from the membrane, and lysis [7]. Caspase-1 [ICE for IL (interleukin)-converting enzyme], a member in the inflammatory caspase family, was the first kind of caspases discovered involved in facilitating pro-IL-1b to form mature IL-1b $[8,9]$. Caspase1-dependent plasma membrane pores require the gradients of cellular ions, contributing to an increase in 
the osmotic pressure, which results in cell swelling and water influx [10]. Caspase-1 dependence, which regulates cell lysis, is a specific characteristic in pyroptosis but not apoptosis [11-13]. The gasdermin family is a major executor in pyroptosis which contains pejvakin(PJVK or DFNB59) and gasdermin-A to gasdermin-E [14]. Inflammasomes participate in the activation of Caspase-1, resulting in GSDMD (gasdermin D) cleavage and the maturation and secreting pro-inflammatory factors, including IL-1B and IL-18 [15]. Except for GSDMD, cleavage of gasdermin proteins of other kinds also induce formation of pores on plasma membrane. Particularly, Caspase-3 participates in the cleavage of GSDME (Gasdermin E) to induce pyroptosis $[16,17]$.

According to the previous studies, it was discovered that pyroptosis had a pivotal part in the development of malignancies and antitumor activities. For instance, recent research found a novel gene signature related to pyroptosis which might be used in the prognosis of Skin Cutaneous Melanoma [18]. Nonetheless, the specific effects of pyroptosis remain to be explored in ccRCC. Thus, this systematic study was conducted to explore the expression difference of genes related to pyroptosis in CCRCC and normal tissues. Meanwhile, a new PRGs prognostic risk signature in ccRCC has been established to predict survival. Furthermore, the phenotypes related to prognosis, and the relationships between the immune microenvironment of tumor and pyroptosis were investigated.

\section{Materials And Methods}

\section{Collection of TCGA Data}

The sequencing data of transcriptome RNA and the clinical data were downloaded (website: https://portal.gdc.cancer.gov/; TCGA database) with a total of $611 \mathrm{ccRCC}$ cases (72 cases of normal samples, and 539 cases of tumor samples). Samples without intact information were excluded.

\section{Identification of DEGs related to pyroptosis}

A total of 52 genes associated with pyroptosis were extracted from prior reviews [19-26] and shown in Table S1. In order to find DEGs associated with pyroptosis between tumor and normal tissues, the "limma"package was applied. Version 11.0 STRING (Search Tool for the Retrieval of Interacting Genes; website: https://string-db.org/) was used to construct PPI network. R programming language and pheatmap package were utilized to acquire heatmaps of DEGs. igraph" and "reshape2" R packages were applied to evaluating the relationship between the selected DEGs.

\section{Consensus Clustering}

To categorize the ccRCC according to consensus clustering, "limma" and "ConsensusClusterPlus" R packages were applied. The relationships between clinical characteristics (OS, overall survival) and clusters were evaluated via using the R package "survival" and chi-square test. The KM (Kaplan-Meier) curves and heatmaps were used to present the results by using R packages "pheatmap", "survival", and "survminer". 


\section{Development of ccRCC Prognostic Models based on PRGs}

The Cox regression analysis (least absolute shrinkage and selection operator, abbreviated as LASSO) was conducted by employing the R package "glmnet" to construct a model of prognosis using candidate genes. The minimum parameters were used to determine the penalty parameter $(\lambda)$. The following equations were used to calculate the RS (risk score): RS equals to $e^{\Sigma i(C o e f i \cdot E x p i)}$, where Expi stands for expression of each retained gene and Coefi for coefficient. PCA (principal component analysis) was performed by applying R package "Rtsne" according to the risk score. The R packages "survival" and "survminer" were applied for KM analyses. And the 1-, 3- and 5-year ROC analyses were performed by using the R package "survivalROC".

\section{Analysis on Prognostic Value of the RS}

The clinical features (stage age, grade, gender, and T and M classification) of the patients were obtained in the TCGA cohort. The RS and these extracted variables were analyzed together in the regression model, with multivariable and univariate Cox regression models used.

\section{Analysis of Gene Sets Enrichment}

In the TCGA cohort, patients with ccRCC were assigned into 2 subgroups based on the median RS. DEGs between the HR (high-risk) group and the LR (low-risk) group were screened based on the criteria (FDR< 0.01 and $|\log 2 \mathrm{FC}| \geq 1.5$ ). According to the DEGs, KEGG and GO analyses were conducted via using the "clusterProfiler" package. The ssGSEA was conducted by using "pheatmap package", "gsva" package to assess activities of the pathways linked to the immune responses after determination of the scores of IIC (infiltration immune cells).

\section{Analysis of TIMER Database}

Comprehensive analysis is conducted using TIMER database (website: https://cistrome.shinyapps.io/timer) and visualization of IIC in over ten thousand tumors of thirty-two types of cancers is facilitated [27]. Six subsets of tumor-infiltrating immune cells (macrophages, dendritic cells, CD8T cells, neutrophils, CD4T cells, and B cells) were contained in TIMER. A new statistical approach was used to assess abundance of the 6 types of IIC in the tumor microenvironment. The genomic, immunological, and clinical features of the tumor were comprehensively investigated by using the TIMER database. In ccRCC, for each hub gene in the risk score model, SCNA (Somatic Copy Number Alterations) module of TIMER tool was used to compare the infiltration between samples containing different SCNA, including high amplification, diploid/normal, deep deletion, arm-level deletion, and armlevel gain [28]. Additionally, the infiltration level in ccRCC samples was harvested using the TIMER database for determination of the relationship with the system of RS.

\section{Statistics}


R software (version 4.1.0) and the packages mentioned above were used for statistical analysis. The logrank test and K-M method were used to perform survival analyses. The significance of prognostic factors was evaluated by using multivariate and univariate Cox regression analyses. In subgroup differential analyses, Kruskal-Wallis test and Wilcoxon rank-sum test were applied. All statistical tests were twosided. $\mathrm{P}<0.05$ was regarded as statistically significant.

\section{Results}

\section{Identification of Differentially Expressed PRGs Between Normal and Tumor Tissues}

The expression levels of 52 genes related to pyroptosis in TCGA (The Cancer Genome Atlas) database were compared between 539 tumor and 72 normal tissues. Ultimately, 44 DEGs were identified. Thirteen genes (NLRP2, TP63, CYCS, CASP9, IL1A, CHMP2B, CHMP4C, CHMP3, IL1B, CHMP7, TIRAP, CASP6, and GSDME) were downregulated while 31 other genes (CHMP2A, IRF2, CHMP6, TP53, GPX4, CASP3, PLCG1, NOD1, GSDMD, CASP8, CHMP4A, IL18, IL6, IRF1, NLRP1, CASP4, BAX, NLRP3, NLRP6, GSDMA, CASP1, GSDMB, NLRC4, PYCARD, NLRP7, GSDMC, NOD2, GZMB, CASP5, AIM2, GZMA) were upregulated in tumor tissue samples. Heatmaps were used to present the RNA expression of these genes in Fig. 1A (blue: a low level of expression; red: a high level of expression).

To further investigate the interactions between these PRGs, PPI (protein-protein interaction) analysis was conducted via applying the STRING platform. The result was illustrated in Fig. 1B. The minimum interaction score required for PPI analysis was 0.9 (the highest confidence). According to the number of nodes, the top 30 hub genes were listed in Fig. 1C. Moreover, except for CHMP4B and HMGB1, DEGs between normal tissues and tumor tissues were shown. The network of correlation including all the genes linked to pyroptosis was demonstrated in Fig. 1D (blue color, negative; red color, positive).

\section{Tumor categorization according to the DEGs linked to pyroptosis}

In order to investigate the relationship of the ccRCC subtypes with the 44 DEGs levels, CCA (consensus clustering analysis) was performed by using the data of 539 ccRCC (Table 1) samples in the cohort of TCGA. When the clustering variable $(k)$ was raised from two to ten, the intragroup correlation reached the peak. Moreover, the intergroup correlation was low when $\mathrm{k}=2$, indicating that $539 \mathrm{ccRCC}$ patients could be well divided into two groups according to 44 DEGs, as shown in Fig. 2A. The clinical characteristics and gene expression profile are presented in a heatmap. After comparing the cluster 1 with cluster 2 , we observed that there were significant differences in clinical stage (stage I-IV), distant metastases (M0-1), Fuhrman grade (G1-G4), and tumor size (T1-T3), whereas the differences in the number of LNM (lymph node metastases), gender and age were not statistically significant (Fig. 2B). We also compared OS of 
the 2 clusters, which was presented in Fig. 2C. OS was significantly poorer in cluster 1 than cluster $2(P<0.001$, Fig. $2 \mathrm{C})$. 
Table 1

Characteristics of patients $(n=537$, here we deleted two duplicate tumor samples).

\begin{tabular}{|ll|}
\hline Variables & Number (\%) \\
\hline Status & \\
\hline Alive & $360(67.04 \%)$ \\
\hline Dead & $177(32.96 \%)$ \\
\hline Gender & \\
\hline Male & $346(64.43 \%)$ \\
\hline Female & $191(35.57 \%)$ \\
\hline Age, years & \\
\hline$>65$ & $185(34.45 \%)$ \\
\hline$<=65$ & $352(65.55 \%)$ \\
\hline Fuhrman grade & \\
\hline Grade 1 & $14(2.61 \%)$ \\
\hline Grade 2 & $230(42.83 \%)$ \\
\hline Grade 3 & $207(38.55 \%)$ \\
\hline Grade 4 & $78(14.52 \%)$ \\
\hline Unknown & $8(1.49 \%)$ \\
\hline Pathological stage & \\
\hline Stage I & $269(50.09 \%)$ \\
\hline Stage II & $57(10.61 \%)$ \\
\hline Stage III & $125(23.28 \%)$ \\
\hline Stage IV & $275(51.21 \%)$ \\
\hline unknown & \\
\hline T stage & $(3.56 .85 \%)$ \\
\hline T1 & \\
\hline T2 & \\
\hline T3 & \\
\hline T4 & \\
\hline
\end{tabular}




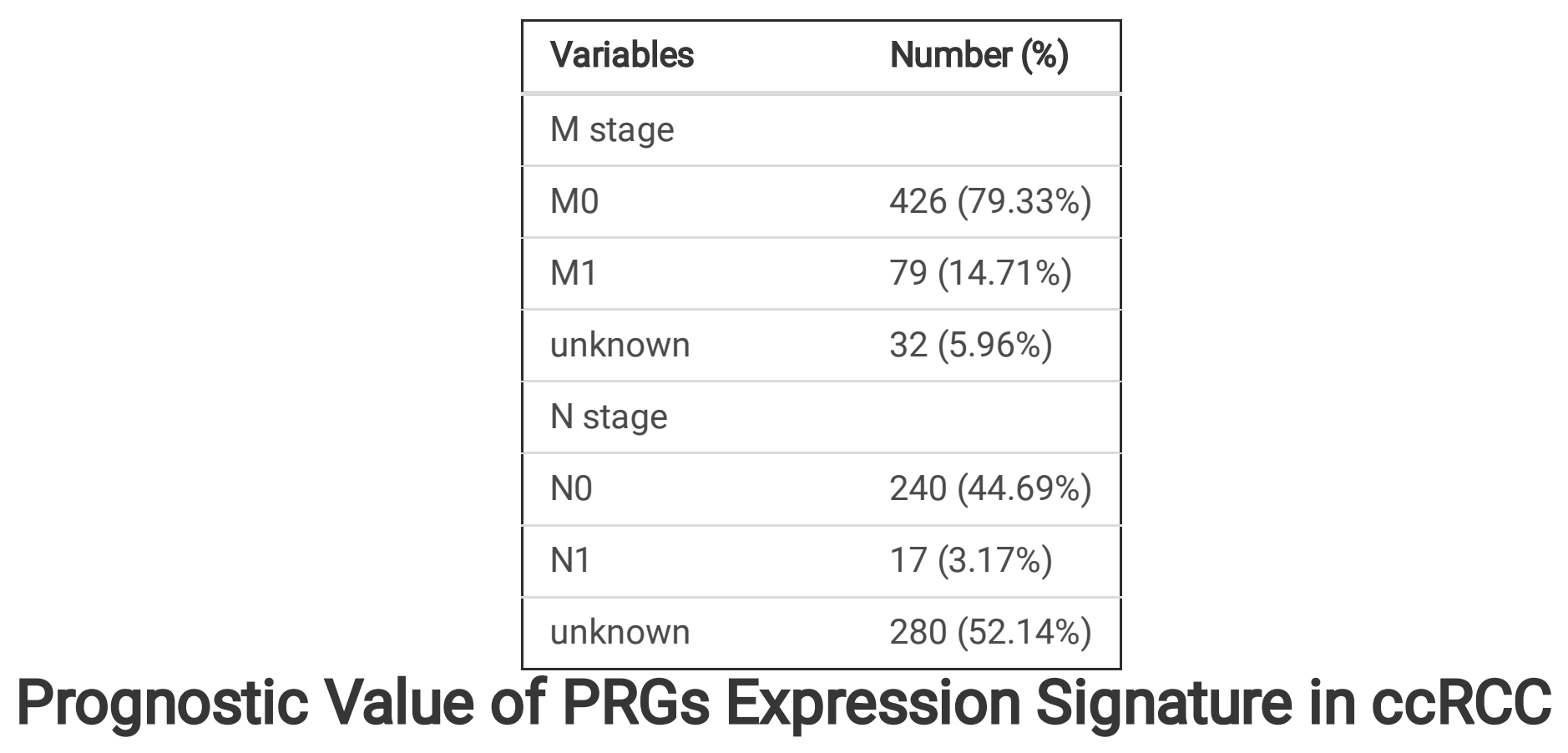

There were 530 ccRCC samples matched with corresponding cases with intact information about survival. We used univariate Cox regression analysis to initially screen for survival-related PRGs, and 8 genes with $\mathrm{P}<0.05$ were used for analysis (Fig. 3A). To screen candidate genes for construction of the model of prognosis, Cox regression analysis (LASSO) was performed. Based on the optimal $\lambda$ value, 6 genes (Fig. 3B, C) and their coefficients (Table 2) were eventually preserved. According to the median score, 530 patients were equally assigned into high- and low-risk subgroups (Fig. 3D). As shown in Fig. $3 E, P C A$ demonstrated that subjects with alternative risks were divided into 2 clusters. The death rate was higher and survival time shorter in the high-risk group compared with the low-risk group (as shown in Fig. $3 F$ ). As shown in Figure 3G, there was significant difference in ccRCC. In the high-risk subgroup, life span was shorter compared with low-risk subgroup $(P<0.001)$. The specificity and sensitivity of the prognostic model were assessed by applying ROC (receiver operating characteristic) curve, and AUC (area under the ROC curve) was $0.706,0.640$ and 0.720 for 5-year, 3-year, and 1-year survival, respectively (Fig. 3H).

Table 2

Construction of a pyroptosis-related signature in cCRCC

\begin{tabular}{|llll|}
\hline Gene name & Coefficients & HR $(95 \% \mathrm{Cl})$ & P value \\
\hline ELANE & 0.003234381 & $1.136(1.043-1.236)$ & 0.003221789 \\
\hline AIM2 & 0.077194693 & $1.256(1.143-1.379)$ & $2.00 \mathrm{E}-06$ \\
\hline GSDMB & 0.267608183 & $1.384(1.236-1.549)$ & $1.82 \mathrm{E}-08$ \\
\hline IL6 & 0.113880538 & $1.174(1.109-1.242)$ & $3.33 \mathrm{E}-08$ \\
\hline NLRP1 & -0.089187177 & $1.263(1.077-1.482)$ & 0.00404868 \\
\hline NOD2 & 0.004289527 & $1.282(1.135-1.447)$ & $6.28 \mathrm{E}-05$ \\
\hline HR, hazard ratio; Cl, confidence interval & \\
\hline
\end{tabular}




\section{Prognostic value of the risk model in ccRCC}

To identify the prognostic value of the risk model, we conducted Kaplan-Meier analysis to confirm whether these genes involved in the construction of risk model were associated with the prognosis of ccRCC. In Fig. 4A, higher levels of ELANE, AIM2, GSDMB, IL6, NLRP1, and NOD2 were positively correlated with poor prognosis. We then analyzed if RS of the gene signature model was a factor predicting prognosis. The results suggested that RS in the cohort of TCGA was a predictive factor of prognosis (HR=3.065, 95\% Cl: 2.295-4.092, Fig. 4B). According to the results of multivariate analysis, after adjustment of confounding factors, RS was a predictive factor for the prognosis of patients with ccRCC in the cohort of TCGA (HR=2.251, 95\% Cl: 1.659-3.055, Fig. 4C). Additionally, for the cohort of TCGA, a heatmap of clinical characteristics was generated (Fig. 4D). It was discovered that there was significant difference in the tumor grade and stage between the subgroups. These findings suggested that the model of prognosis based on PRGs was robust and independent in the prediction of ccRCC prognosis.

\section{Identifying the Prognostic Model-Related BP (Biological Processes)}

It was of great importance to find out what BP were affected by the model of prognostic risk in the role of prediction. The"limma" R package was utilized to obtain the differentially expressed genes: $F D R<0.01$, $|\log 2 \mathrm{FC}| \geq 1.5$. As a result, a total of $381 \mathrm{DEGs}$ in the cohort of TCGA were selected.378 of 381 genes in the high-risk group were over-expressed, and reduced expression was indicated in the remaining 3 genes (Table S3). KEGG pathway analysis and GO enrichment analysis were conducted by using the selected DEGs (Fig. 5). Of interest, the most enriched biological processes were closely related to immune responses, inflammatory cell chemotaxis, and chemokine-mediated signalling pathways, including B cell regulated immunity, responses of humoral immune, CXCR chemokine receptor binding, and TNF/ NF-kappa B/ IL-17 pathways. These findings suggested that the model of prognostic risk based on PRGs was associated with immune responses.

\section{Evaluation of Immune Cell Infiltration between subgroups}

According to the above findings, it was proposed that the functions of PRGs in the prediction of ccRCC prognosis might be associated with the immune microenvironment. The relationship was evaluated between the infiltration of immune cells and prognosis-related genes. The changes of infiltration were explored by using the samples with copy number alteration of ELANE, AIM2, GSDMB, IL6, NIRP1, and NOD2, respectively. The results showed that copy number of these prognosis-related PRGs was associated with the immune microenvironment in ccRCC. Mutants of AIM2, ELANE, GSDMB, NLRP1, and NOD2 inhibited the infiltration of some kinds of immune cells (Fig. 6A-F). Then, the immune estimation dataset was downloaded from the TIMER database and the correlation was analyzed between the infiltration of 6 types of immune cell (macrophages, dendritic cells, neutrophils, B cells, CD $8+T$ cells, and CD 4+T cells) and the risk score of the prognostic model. The data suggested that the risk score of the PRGs-related prognosis model was positively correlated with immune infiltration (Fig. 7A-F). 
The activities of thirteen pathways related to immune responses and enrichment scores of sixteen kinds of immune cells were then compared between the HR group and the LR group in the cohort of TCGA using SSGSEA (single-sample gene set enrichment analysis). The results revealed that infiltration was increased in the HR group, especially DCs, Th cells (Th2, Tfh, and Th1 cells), CD8+ T cells, Macrophages, B cells, Treg cells, pDCs, TILs (tumor-infiltration lymphocytes) and Neutrophils, compared to the LR group, as shown in Fig. 8A. In Fig. 8B, it was illustrated that all the13 immune pathways in the high-risk group were more active compared with the low-risk group.

The study design and grouping are shown in Fig. 9.

\section{Discussion}

Pyroptosis is a newly recognized kind of programmed cell death, which has a double-edged role in the progression of malignancy and mechanisms of treatment. Inflammatory cytokines are secreted in pyroptosis and normal cells are stimulated, contributing to the progression of cancer [19]. Meanwhile, pyroptosis can enhance cellular death in malignancy, which makes pyrolysis a possible therapeutic and prognostic target for malignancy [29]. However, how the genes related to pyroptosis interact with each other and whether they have impact on the survival time in ccRCC remain unrevealed.

In this research, the mRNA expression of 52 currently known PRGs were first discovered in normal and cCRCC tissues. It was found that 44 of them were differentially expressed. What makes sense is that the 2 clusters in the consensus clustering analysis according to the pyroptosis-related DEGs showed significant differences in clinical characteristics. This implied that pyroptosis in cancer tissues was different in ccRCC, which resulted in different OS.

To further evaluate the prognostic value of the regulators related to pyroptosis, a 6-gene risk signature was constructed. OS in cases of alternative subgroups was different. The functional analyses presented that the DEGs between the high- and low-risk groups showed significant differences in immune-related pathways, consistent with our expectations. Pyroptosis could contribute to the accumulation of various inflammatory factors, which was also resulted from the activation of inflammasomes [6, 30]. Additionally, we also enriched Cytokine, NF-kappa B, IL-17, Ras and TNF signaling pathways which were closely associated with the development of RCC.

Meanwhile, the pathway activation and immune infiltration were compared between the HR group and the LR group, and the HR group showed increased activities of pathways related to immune responses and elevated levels of IIC in comparison with the LR group. Enhanced infiltration of immune cells was related to poor prognosis, which was consistent with previous studies[31]. Another key result in our research was that the above 6 prognostic genes related to pyroptosis had a significant correlation with immune infiltration, which further proved the fact that pyroptosis had an essential part in the immune microenvironment of tumor. 
The current study found a signature of 6 genes linked to pyroptosis (ELANE, AIM2, GSDMB, IL6, NIRP1, and NOD2) and discovered that it could play a role in predicting OS of cCRCC patients. AIM2 (Absent in melanoma 2) was first found in melanoma with decreased expression[21]. AIM2 is a member of the IFNinducible PYHIN (pyrin and HIN200 domain-containing) family, and works as a cytoplasmic senor for DNA which can connect with dsDNA (double-stranded DNA) [32]. AIM2 can activate CASP-1 via junctional proteins regulated by ASC to facilitate secretion and maturation of IL-18 and IL-1 $\beta$, thus promoting pyroptosis[33]. Previous research demonstrated that AIM2 worked as a suppressor in multiple kinds of tumors such as prostate cancer [34], colon cancer [35], melanoma [36], melanoma [37], and breast cancer but as a promoter in NSCLC (non-small cell lung cancer) [38], OSCC (oral squamous cell carcinoma) [39], and HPV (human papillomavirus)-associated cervical cancer [40]. Therefore, AIM2 might had different effects in different tumors. In this research, the expression of AIM2 was significantly increased in tumor tissues in comparison with normal ones. Furthermore, an increased expression level of AIM2 was closely related to poor survival and gene mutations in AIM2 could ameliorate infiltration of immune cells. Thus, it was suggested that AIM2 was more like a pro-oncogene. The molecular mechanisms of AIM2 in the development of ccRCC currently remained unrevealed and our findings in AIM2 might provide a new insight into further research.

GSDMB (Gasdermin B) was the gene most linked to RS in the prognostic model, prompting that GSDMB might be highly involved in ccRCC. According to previous research on human malignancies, it was found that GSDMB was upregulated in tumor tissues, including breast, uterine, gastric, and cervical cancers [41]. It was demonstrated that GSDMB was located in amplicons, the genomic regions frequently amplified in cancer development [42]. Thus, GSDMB might participate in cancer development and metastasis. GSDMB can be cleaved into two fragments by caspasa-1. One cleavage form is the N-terminal of GSDMB protein with a molecular weight of $20 \mathrm{kDa}$. Cell pyroptosis can be caused by the secretion of the $\mathrm{N}$ terminal domain. On the contrary, the full-length $\mathrm{N}$-terminal domain and $\mathrm{C}$-terminal fragment would not cause cell pyroptosis $[43,44]$. In general, GSDMB may be the downstream protein of pyroptosis pathway. The key is whether some factors can trigger the upstream mechanism of GSDMB and cause pyroptosis. At the same time, the specific mechanism of GSDMB in cCRCC is not clear. The increased level of GSDMB in cCRCC is related to poor prognosis. This finding may provide help for the study of tumor treatment targets.

ELANE (neutrophil elastase gene) is a main serine protease produced by neutrophils, which can activate proinflammatory cytokines including IL-1 $\beta, I L-18$, and TNF-a[45, 46], which are regarded as promoters for pyroptosis. Kambara et al.[47] presented that ELANE could cleave and activate GSDMD and subsequently induce pyroptosis in neutrophils. The ELANE expression level in the HR group was remarkably increased compared with the LR group, but paradoxically, the neutrophil infiltration score was incredibly higher compared with the LR group. These findings may be resulted from many complex factors driving the difference of gene expression between alternative tissues, particularly levels of genes and PRGs linked to inflammation, such as the proportion of infiltrated immune cells and the differentiation of ccRCC $[48,49]$. Such factors might not influence application of PRG expression signature in diagnosing and predicting ccRCC prognosis. The relationship between the expression of pyroptosis genes and infiltration of immune 
cells, ccRCC differentiation status, and other factors requires further investigation, which may provide new enlightenment for predicting the diagnosis and prognosis of ccRCC.

NLRP1 (NLR family, pyrin domain containing1), a bipartite adaptor protein, is considered as apoptosisassociated speck-like protein with a ASC (caspase-recruitment domain). NLRP1 can promote the recruitment process of pro-caspase- 1 to the complex of inflammasomes [50]. GSDMD can be cleaved by active caspase-1, allowing the N-terminal domain of GSDMD to form pores in the plasma membrane, and then triggers the pyroptosis mechanism[10, 15, 51, 52]. NOD2 (nucleotide-binding oligomerization domain-containing protein 2), which can initiate NF-KB (nuclear factor-kB)-dependent and MAPK (mitogen-activated protein kinase)-dependent gene transcription. For macrophages, NOD2 could be used as its promoter to induce the activation of inflammatory bodies[53]. In our study, NOD2 was highly expressed in the HR group, which might be one of the reasons why macrophages obtained higher scores in the HR group compared with the LR group. At the same time, the activation effect of NOD2 on NF-KB pathway might make this pathway more enriched in KEGG analysis. Interestingly, as one of the genes in the risk prognostic model, activated NF-kB pathway was able to increase the amount of mRNA for IL-6 [54]. In advanced metastatic breast cancer cells, over-activated NF-kB promoted chromatin accessibility of the IL- 6 promoter region and enhanced transcription of the IL- 6 gene[55]. Appropriate expression of IL- 6 is of great importance for human immune defense, but sustained production of IL- 6 has a pivotal role in the occurrence of multiple inflammation-related diseases and cancer[56]. Whereas in our study, IL-6 was significantly highly expressed in the HR group, we speculated that NOD2 might act as an upstream initiator of IL-6 to promote IL-6 expression by activating the NF-kB pathway.

The purpose of our research was to categorize cases with ccRCC into different subtypes, screen DEGs, set up a model of prognosis, and connect pyroptosis with the prognosis. Though we conducted multi-angle and multi-omics validation, there were still limitations in this study. All the analyses were performed using the TCGA KIRC cohort, and GEO cohort was recommended to verify them. Additionally, in-vivo and in-vitro experiments are required to further verify our findings. Pyroptosis, particularly the mechanism in ccRCC, is not sufficiently investigated. We initially investigated the prognostic value of the 6 genes related to pyroptosis in the risk prognostic model, which provided theoretical support for future studies.

\section{Conclusions}

In conclusion, a comprehensive and systematic bioinformatics analysis was performed and we found a prognostic gene signature related to pyroptosis including six genes (ELANE, AIM2, GSDMB, IL6, NIRP1, and NOD2) for ccRCC patients. Moreover, the risk score in the prognostic model according to the 6 PRGs was an independent prognostic factor of cases with ccRCC, which was associated with the immune microenvironment.

\section{Declarations}

\section{Authors' contributions}


Conceptualization, Z.-Y.L. and Z.-N.X.; methodology, Z.-Y.L. and Y.-P.Y.; software, Z.-Y.L. and B.-W.W.; validation, L.-C.C., W.-G.J. and T.-D.W.; formal analysis, Z.-Y.L. and Z.-N.X.; investigation, Y.-P.Y. and W.-X.; resources, Z.-Y.L. and Z.-N.X.; data curation, Z.-Y.L.; writing-original draft preparation, Z.-Y.L and X.-Y.W.; writing-review and editing, Z.-Y.L., C.-Y.W. and C.-Z.; visualization, Z.-Y.L., P.-Z. and W.-H.Y.; supervision, Z.Y.L., C.-Y.W. and C.-Z.; project administration, C.-Y.W. and C.-Z.; funding acquisition, C.-Y.W. and C.-Z. All authors have read and agreed to the published version of the manuscript.

\section{Funding}

This research was funded by the Outstanding Young Medical Talents Training Program of the First Affiliated Hospital of Harbin Medical University, China, grant number HYD2020JQ0020, and the National Natural Science Foundation of China, grant number 81872084.

\section{Availability of data and materials}

In the study, diferent web-based datasets were used for data analysis. The web links to all the original data sources were listed as below: The sequencing data of transcriptome RNA and the clinical data were downloaded from The Cancer Genome Atlas Program (TCGA) (https://portal.gdc.cancer.gov/) data portal. All data generated from the analysis process of this study are available from the corresponding author on reasonable request.

\section{Acknowledgments}

We would like to acknowledge the TCGA network for providing data. We thank contributions from TIMER and STRING platforms. In addition, we are very grateful to Professor Wang and Professor Zhang for their teaching during the postgraduate study.

\section{Ethics approval and consent to participate}

The data used in this study were all obtained from the TCGA public database, and for public data use, all methods were performed in accordance with relevant guidelines and regulations.

\section{Consent for publication}

Not applicable.

\section{Competing interests}

The authors declare no conflict of interest.

\section{Author details}

a Department of Urology, The First Affiliated Hospital of Harbin Medical University, Harbin 150001, China.

${ }^{b}$ Department of Urology, The Fourth Affiliated Hospital Zhejiang University School of Medicine, Yiwu City 


\section{References}

1. Clark DJ, Dhanasekaran SM, Petralia F, Pan J, Song X, Hu Y, et al. Integrated Proteogenomic Characterization of Clear Cell Renal Cell Carcinoma. Cell. 2020;180:207. doi:10.1016/j.cell.2019.12.026.

2. Bray F, Ferlay J, Soerjomataram I, Siegel RL, Torre LA, Jemal A. Global cancer statistics 2018 : GLOBOCAN estimates of incidence and mortality worldwide for 36 cancers in 185 countries. CA Cancer J Clin. 2018;68:394-424. doi:10.3322/caac.21492.

3. Ferlay J, Colombet M, Soerjomataram I, Mathers C, Parkin DM, Pineros M, et al. Estimating the global cancer incidence and mortality in 2018: GLOBOCAN sources and methods. Int J Cancer. 2019;144:1941-1953. doi:10.1002/ijc.31937.

4. Ljungberg B, Albiges L, Abu-Ghanem Y, Bensalah K, Dabestani S, Fernandez-Pello S, et al. European Association of Urology Guidelines on Renal Cell Carcinoma: The 2019 Update. Eur Urol. 2019;75:799-810. doi:10.1016/j.eururo.2019.02.011.

5. Kovacs SB, Miao EA. Gasdermins: Effectors of Pyroptosis. Trends Cell Biol. 2017;27:673-684. doi:10.1016/j.tcb.2017.05.005.

6. Bergsbaken T, Fink SL, Cookson BT. Pyroptosis: host cell death and inflammation. Nat Rev Microbiol. 2009;7:99-109. doi:10.1038/nrmicro2070.

7. Miao EA, Rajan JV, Aderem A. Caspase-1-induced pyroptotic cell death. Immunol Rev. 2011;243:206214. doi:10.1111/j.1600-065X.2011.01044.x.

8. Thornberry NA, Bull HG, Calaycay JR, Chapman KT, Howard AD, Kostura MJ, et al. A novel heterodimeric cysteine protease is required for interleukin-1 beta processing in monocytes. Nature. 1992;356:768-774. doi:10.1038/356768a0.

9. Cerretti DP, Kozlosky CJ, Mosley B, Nelson N, Van Ness K, Greenstreet TA, et al. Molecular cloning of the interleukin-1 beta converting enzyme. Science. 1992;256:97-100. doi:10.1126/science.1373520.

10. Fink SL, Cookson BT. Caspase-1-dependent pore formation during pyroptosis leads to osmotic lysis of infected host macrophages. Cell Microbiol. 2006;8:1812-1825. doi:10.1111/j.14625822.2006.00751.x.

11. Kuida K, Lippke JA, Ku G, Harding MW, Livingston DJ, Su MS, et al. Altered cytokine export and apoptosis in mice deficient in interleukin-1 beta converting enzyme. Science. 1995;267:2000-2003. doi:10.1126/science.7535475.

12. Fink SL, Bergsbaken T, Cookson BT. Anthrax lethal toxin and Salmonella elicit the common cell death pathway of caspase-1-dependent pyroptosis via distinct mechanisms. Proc Natl Acad Sci U S A. 2008;105:4312-4317. doi:10.1073/pnas.0707370105.

13. Bergsbaken T, Cookson BT. Macrophage activation redirects yersinia-infected host cell death from apoptosis to caspase-1-dependent pyroptosis. PLoS Pathog. 2007;3:e161. 
doi:10.1371/journal.ppat.0030161.

14. Broz $P$, Pelegrin $P$, Shao $F$. The gasdermins, a protein family executing cell death and inflammation. Nat Rev Immunol. 2020;20:143-157. doi:10.1038/s41577-019-0228-2.

15. Shi J, Zhao $Y$, Wang $K$, Shi $X$, Wang $Y$, Huang $H$, et al. Cleavage of GSDMD by inflammatory caspases determines pyroptotic cell death. Nature. 2015;526:660-665. doi:10.1038/nature15514.

16. Ding J, Wang K, Liu W, She Y, Sun Q, Shi J, et al. Pore-forming activity and structural autoinhibition of the gasdermin family. Nature. 2016;535:111-116. doi:10.1038/nature18590.

17. Rogers C, Fernandes-Alnemri T, Mayes L, Alnemri D, Cingolani G, Alnemri ES. Cleavage of DFNA5 by caspase-3 during apoptosis mediates progression to secondary necrotic/pyroptotic cell death. Nat Commun. 2017;8:14128. doi:10.1038/ncomms14128.

18. Ju A, Tang J, Chen S, Fu Y, Luo Y. Pyroptosis-Related Gene Signatures Can Robustly Diagnose Skin Cutaneous Melanoma and Predict the Prognosis. Front Oncol. 2021;11:709077. doi:10.3389/fonc.2021.709077.

19. Karki R, Kanneganti TD. Diverging inflammasome signals in tumorigenesis and potential targeting. Nat Rev Cancer. 2019;19:197-214. doi:10.1038/s41568-019-0123-y.

20. Xia X, Wang X, Cheng Z, Qin W, Lei L, Jiang J, et al. The role of pyroptosis in cancer: pro-cancer or pro-"host"? Cell Death Dis. 2019;10:650. doi:10.1038/s41419-019-1883-8.

21. Wang B, Yin Q. AIM2 inflammasome activation and regulation: A structural perspective. J Struct Biol. 2017;200:279-282. doi:10.1016/j.jsb.2017.08.001.

22. Man SM, Kanneganti TD. Regulation of inflammasome activation. Immunol Rev. 2015;265:6-21. doi:10.1111/imr.12296.

23. Malik A, Kanneganti TD. Inflammasome activation and assembly at a glance. J Cell Sci. 2017;130:3955-3963. doi:10.1242/jcs.207365.

24. Shi J, Gao W, Shao F. Pyroptosis: Gasdermin-Mediated Programmed Necrotic Cell Death. Trends Biochem Sci. 2017;42:245-254. doi:10.1016/j.tibs.2016.10.004.

25. Rathinam VA, Fitzgerald KA. Inflammasome Complexes: Emerging Mechanisms and Effector Functions. Cell. 2016;165:792-800. doi:10.1016/j.cell.2016.03.046.

26. Fang Y, Tian S, Pan Y, Li W, Wang Q, Tang Y, et al. Pyroptosis: A new frontier in cancer. Biomed Pharmacother. 2020;121:109595. doi:10.1016/j.biopha.2019.109595.

27. Li T, Fan J, Wang B, Traugh N, Chen Q, Liu JS, et al. TIMER: A Web Server for Comprehensive Analysis of Tumor-Infiltrating Immune Cells. Cancer Res. 2017;77:e108-e110. doi:10.1158/0008-5472.CAN-170307.

28. Mermel CH, Schumacher SE, Hill B, Meyerson ML, Beroukhim R, Getz G. GISTIC2.0 facilitates sensitive and confident localization of the targets of focal somatic copy-number alteration in human cancers. Genome Biol. 2011;12:R41. doi:10.1186/gb-2011-12-4-r41.

29. Ruan J, Wang S, Wang J. Mechanism and regulation of pyroptosis-mediated in cancer cell death. Chem Biol Interact. 2020;323:109052. doi:10.1016/j.cbi.2020.109052. 
30. Schroder K, Tschopp J. The inflammasomes. Cell. 2010;140:821-832. doi:10.1016/j.cell.2010.01.040.

31. Diaz-Montero CM, Rini BI, Finke JH. The immunology of renal cell carcinoma. Nat Rev Nephrol. 2020;16:721-735. doi:10.1038/s41581-020-0316-3.

32. Hornung V, Ablasser A, Charrel-Dennis M, Bauernfeind F, Horvath G, Caffrey DR, et al. AIM2 recognizes cytosolic dsDNA and forms a caspase-1-activating inflammasome with ASC. Nature. 2009;458:514-518. doi:10.1038/nature07725.

33. Kumari P, Russo AJ, Shivcharan S, Rathinam VA. AIM2 in health and disease: Inflammasome and beyond. Immunol Rev. 2020;297:83-95. doi:10.1111/imr.12903.

34. Ponomareva L, Liu H, Duan X, Dickerson E, Shen H, Panchanathan R, et al. AIM2, an IFN-inducible cytosolic DNA sensor, in the development of benign prostate hyperplasia and prostate cancer. Mol Cancer Res. 2013;11:1193-1202. doi:10.1158/1541-7786.MCR-13-0145.

35. DeYoung KL, Ray ME, Su YA, Anzick SL, Johnstone RW, Trapani JA, et al. Cloning a novel member of the human interferon-inducible gene family associated with control of tumorigenicity in a model of human melanoma. Oncogene. 1997;15:453-457. doi:10.1038/sj.onc.1201206.

36. Dihlmann S, Tao S, Echterdiek F, Herpel E, Jansen L, Chang-Claude J, et al. Lack of Absent in Melanoma 2 (AIM2) expression in tumor cells is closely associated with poor survival in colorectal cancer patients. Int J Cancer. 2014;135:2387-2396. doi:10.1002/ijc.28891.

37. Chen IF, Ou-Yang F, Hung JY, Liu JC, Wang H, Wang SC, et al. AIM2 suppresses human breast cancer cell proliferation in vitro and mammary tumor growth in a mouse model. Mol Cancer Ther. 2006;5:17. doi:10.1158/1535-7163.MCT-05-0310.

38. Milutin Gasperov N, Farkas SA, Nilsson TK, Grce M. Epigenetic activation of immune genes in cervical cancer. Immunol Lett. 2014;162:256-257. doi:10.1016/j.imlet.2014.09.019.

39. Kondo Y, Nagai K, Nakahata S, Saito Y, Ichikawa T, Suekane A, et al. Overexpression of the DNA sensor proteins, absent in melanoma 2 and interferon-inducible 16, contributes to tumorigenesis of oral squamous cell carcinoma with p53 inactivation. Cancer Sci. 2012;103:782-790. doi:10.1111/j.1349-7006.2012.02211.x.

40. Kong $H$, Wang $Y$, Zeng $X$, Wang $Z$, Wang $H$, Xie W. Differential expression of inflammasomes in lung cancer cell lines and tissues. Tumour Biol. 2015;36:7501-7513. doi:10.1007/s13277-015-3473-4.

41. Das S, Miller M, Broide DH. Chromosome 17q21 Genes ORMDL3 and GSDMB in Asthma and Immune Diseases. Adv Immunol. 2017;135:1-52. doi:10.1016/bs.ai.2017.06.001.

42. Komiyama H, Aoki A, Tanaka S, Maekawa H, Kato Y, Wada R, et al. Alu-derived cis-element regulates tumorigenesis-dependent gastric expression of GASDERMIN B (GSDMB). Genes Genet Syst. 2010;85:75-83. doi:10.1266/ggs.85.75.

43. Panganiban RA, Sun M, Dahlin A, Park HR, Kan M, Himes BE, et al. A functional splice variant associated with decreased asthma risk abolishes the ability of gasdermin $B$ to induce epithelial cell pyroptosis. J Allergy Clin Immunol. 2018;142:1469-1478 e1462. doi:10.1016/j.jaci.2017.11.040. 
44. Shi P, Tang A, Xian L, Hou S, Zou D, Lv Y, et al. Loss of conserved Gsdma3 self-regulation causes autophagy and cell death. Biochem J. 2015;468:325-336. doi:10.1042/BJ20150204.

45. Mirea AM, Stienstra R, Kanneganti TD, Tack CJ, Chavakis T, Toonen EJM, et al. Mice Deficient in the IL-1 beta Activation Genes Prtn3, Elane, and Casp1 Are Protected Against the Development of ObesityInduced NAFLD. Inflammation. 2020;43:1054-1064. doi:10.1007/s10753-020-01190-4.

46. Fu Z, Akula S, Thorpe M, Hellman L. Potent and Broad but not Unselective Cleavage of Cytokines and Chemokines by Human Neutrophil Elastase and Proteinase 3. Int J Mol Sci. 2020;21. doi:10.3390/ijms21020651.

47. Kambara H, Liu F, Zhang X, Liu P, Bajrami B, Teng Y, et al. Gasdermin D Exerts Anti-inflammatory Effects by Promoting Neutrophil Death. Cell Rep. 2018;22:2924-2936. doi:10.1016/j.celrep.2018.02.067.

48. Erkes DA, Cai W, Sanchez IM, Purwin TJ, Rogers C, Field CO, et al. Mutant BRAF and MEK Inhibitors Regulate the Tumor Immune Microenvironment via Pyroptosis. Cancer Discov. 2020;10:254-269. doi:10.1158/2159-8290.CD-19-0672.

49. Rathore M, Girard C, Ohanna M, Tichet M, Ben Jouira R, Garcia E, et al. Cancer cell-derived long pentraxin 3 (PTX3) promotes melanoma migration through a toll-like receptor 4 (TLR4)/NF-kappaB signaling pathway. Oncogene. 2019;38:5873-5889. doi:10.1038/s41388-019-0848-9.

50. Fernandes-Alnemri T, Wu J, Yu JW, Datta P, Miller B, Jankowski W, et al. The pyroptosome: a supramolecular assembly of ASC dimers mediating inflammatory cell death via caspase-1 activation. Cell Death Differ. 2007;14:1590-1604. doi:10.1038/sj.cdd.4402194.

51. Kayagaki N, Stowe IB, Lee BL, O'Rourke K, Anderson K, Warming S, et al. Caspase-11 cleaves gasdermin D for non-canonical inflammasome signalling. Nature. 2015;526:666-671. doi:10.1038/nature15541.

52. He WT, Wan H, Hu L, Chen P, Wang X, Huang Z, et al. Gasdermin D is an executor of pyroptosis and required for interleukin-1 beta secretion. Cell Res. 2015;25:1285-1298. doi:10.1038/cr.2015.139.

53. Philpott DJ, Sorbara MT, Robertson SJ, Croitoru K, Girardin SE. NOD proteins: regulators of inflammation in health and disease. Nat Rev Immunol. 2014;14:9-23. doi:10.1038/nri3565.

54. Inouye S, Fujimoto M, Nakamura T, Takaki E, Hayashida N, Hai T, et al. Heat shock transcription factor 1 opens chromatin structure of interleukin- 6 promoter to facilitate binding of an activator or a repressor. J Biol Chem. 2007;282:33210-33217. doi:10.1074/jbc.M704471200.

55. Ndlovu MN, Van Lint C, Van Wesemael K, Callebert P, Chalbos D, Haegeman G, et al. Hyperactivated NF-\{kappa\}B and AP-1 transcription factors promote highly accessible chromatin and constitutive transcription across the interleukin- 6 gene promoter in metastatic breast cancer cells. Mol Cell Biol. 2009;29:5488-5504. doi:10.1128/MCB.01657-08.

56. Tanaka T, Narazaki M, Masuda K, Kishimoto T. Regulation of IL-6 in Immunity and Diseases. Adv Exp Med Biol. 2016;941:79-88. doi:10.1007/978-94-024-0921-5_4.

\section{Figures}


A

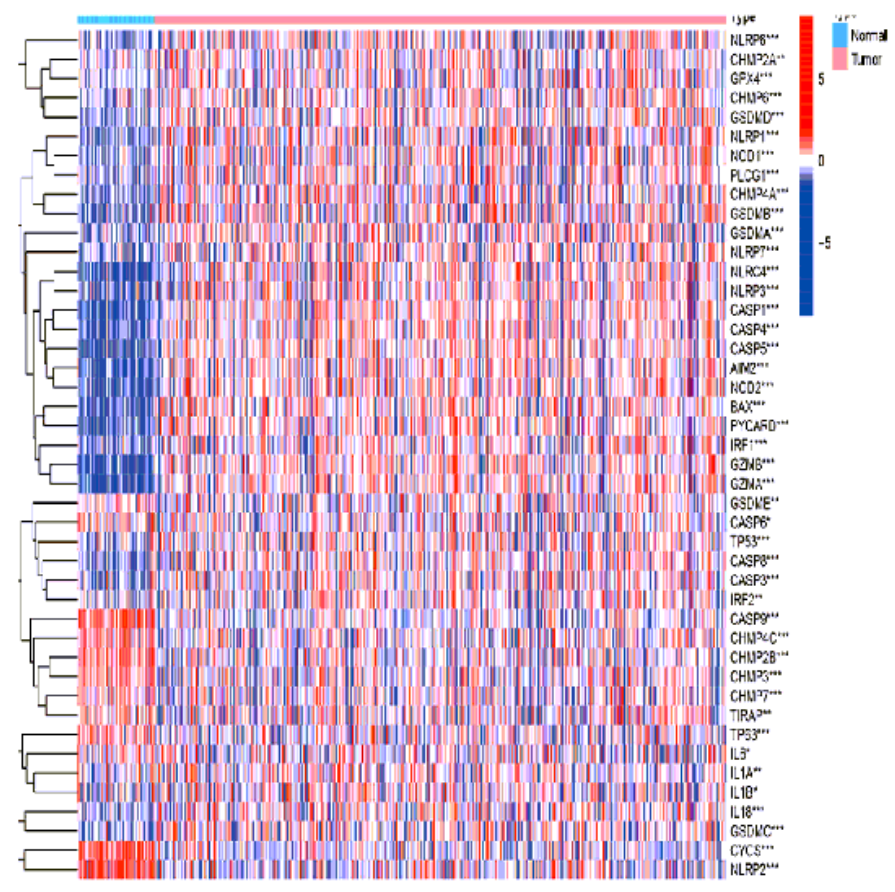

$\mathrm{C}$

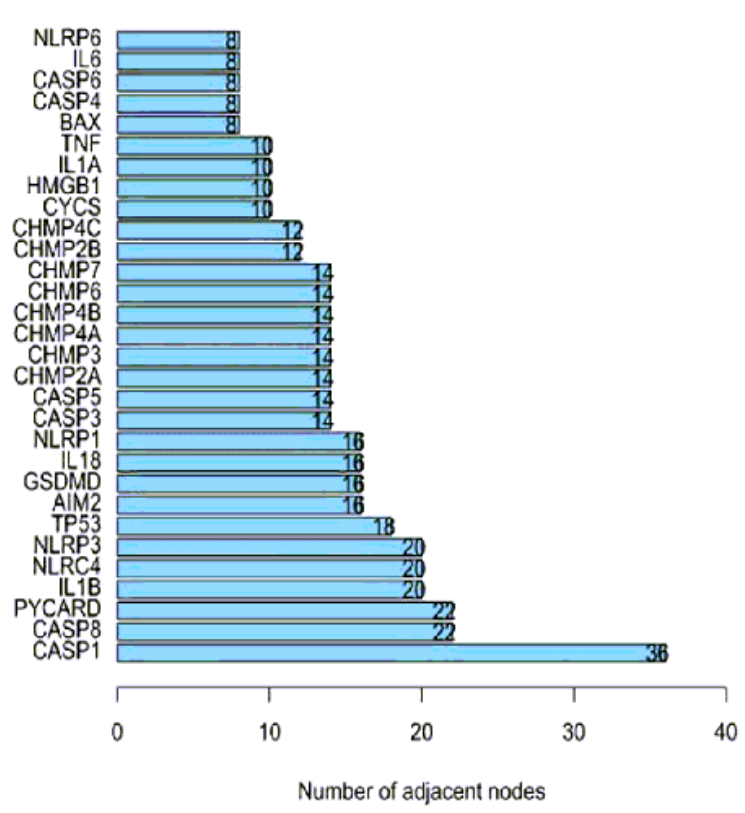

B
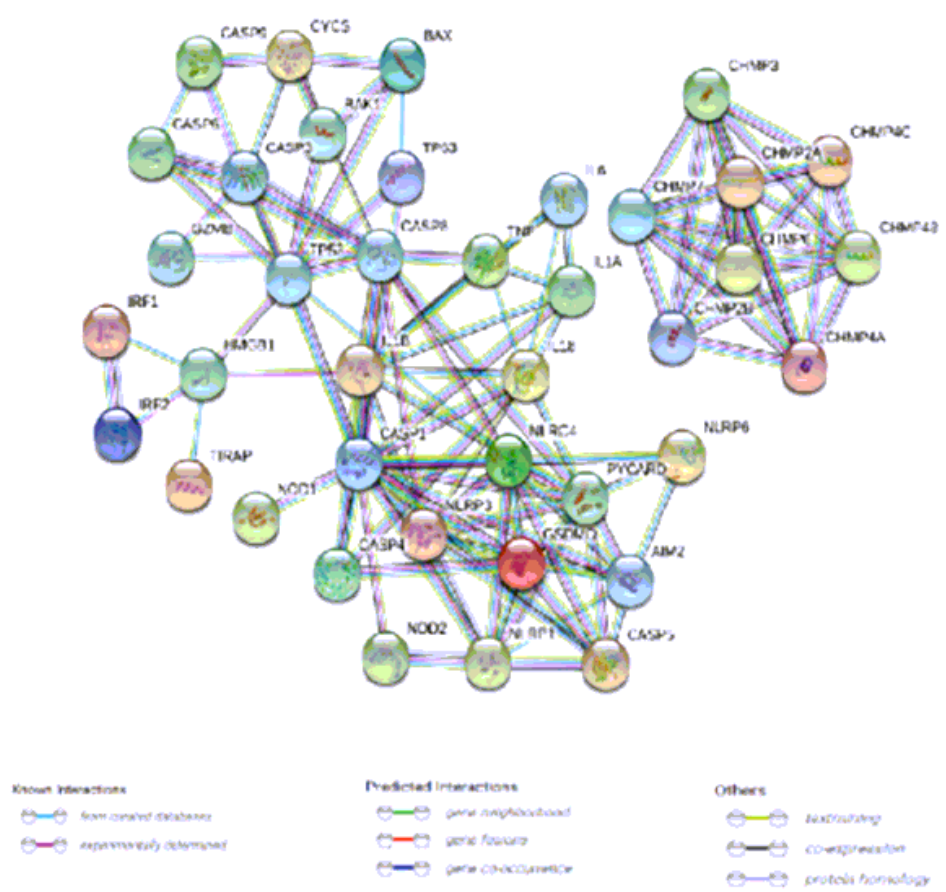

$\mathrm{D}$

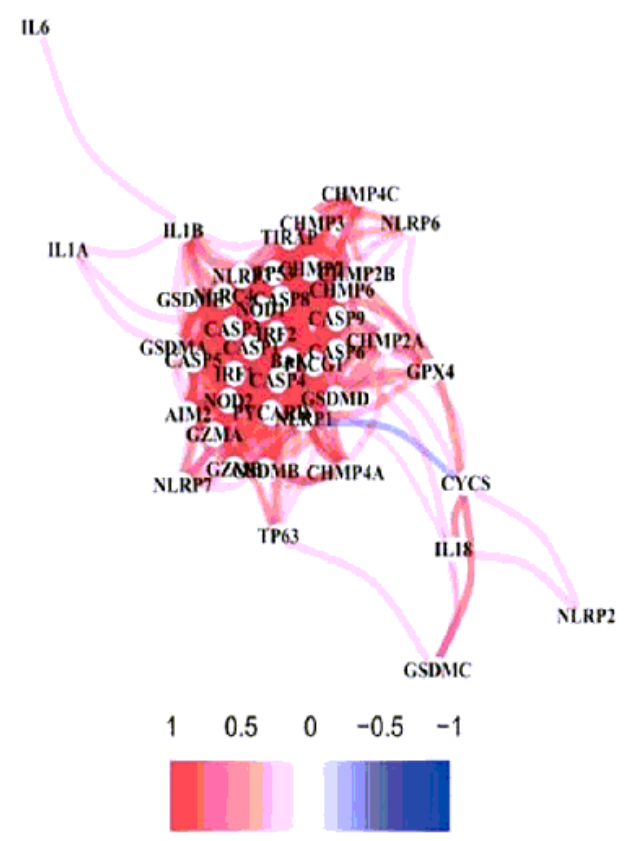

Figure 1

Expressions and interactions of the 44 pyroptosis-linked genes. A Heatmap (blue: low level of expression; red: high level of expression) of the genes linked to pyroptosis between the tumor ( $T$, brilliant red) and the normal ( $N$, brilliant blue) tissues. ${ }^{* *} \mathrm{P}<0.001,{ }^{*} \mathrm{P}<0.01,{ }^{*} \mathrm{P}<0.05$. B The interaction between the genes linked to pyroptosis indicated by PPI network (the score of interaction is 0.9 ). C Hub genes ranked by node number. D The correlation network of the genes related to pyroptosis (blue color, negative; red color, positive). The color depth represents relevance strength. 
A

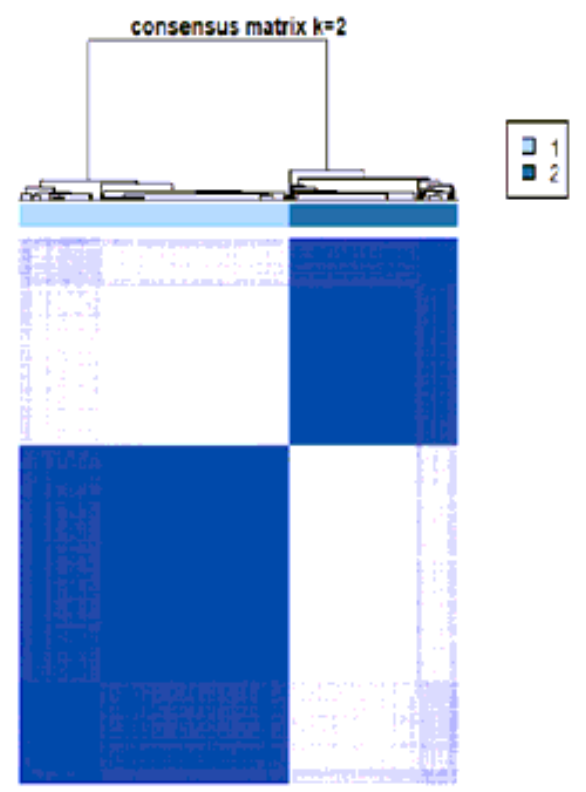

$\mathrm{C}$

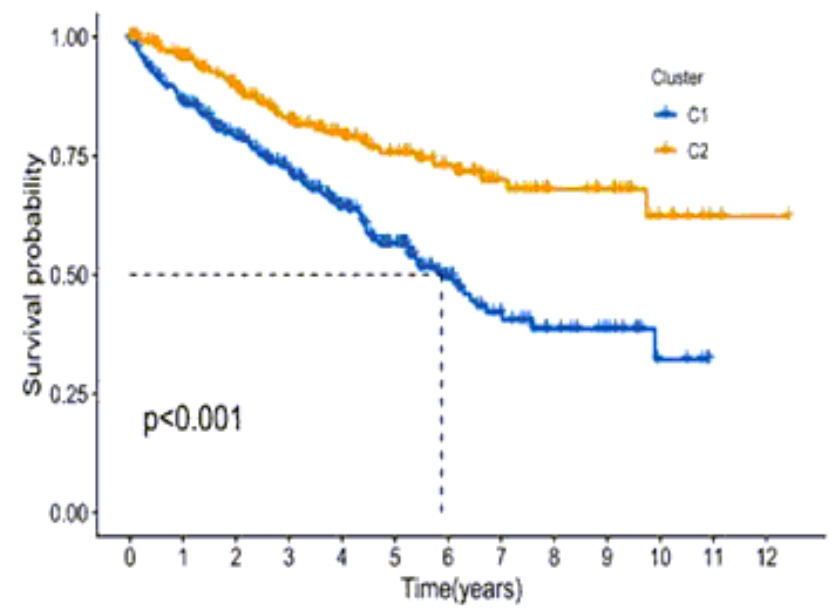

Number at risk

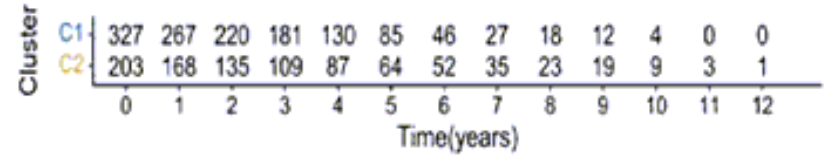

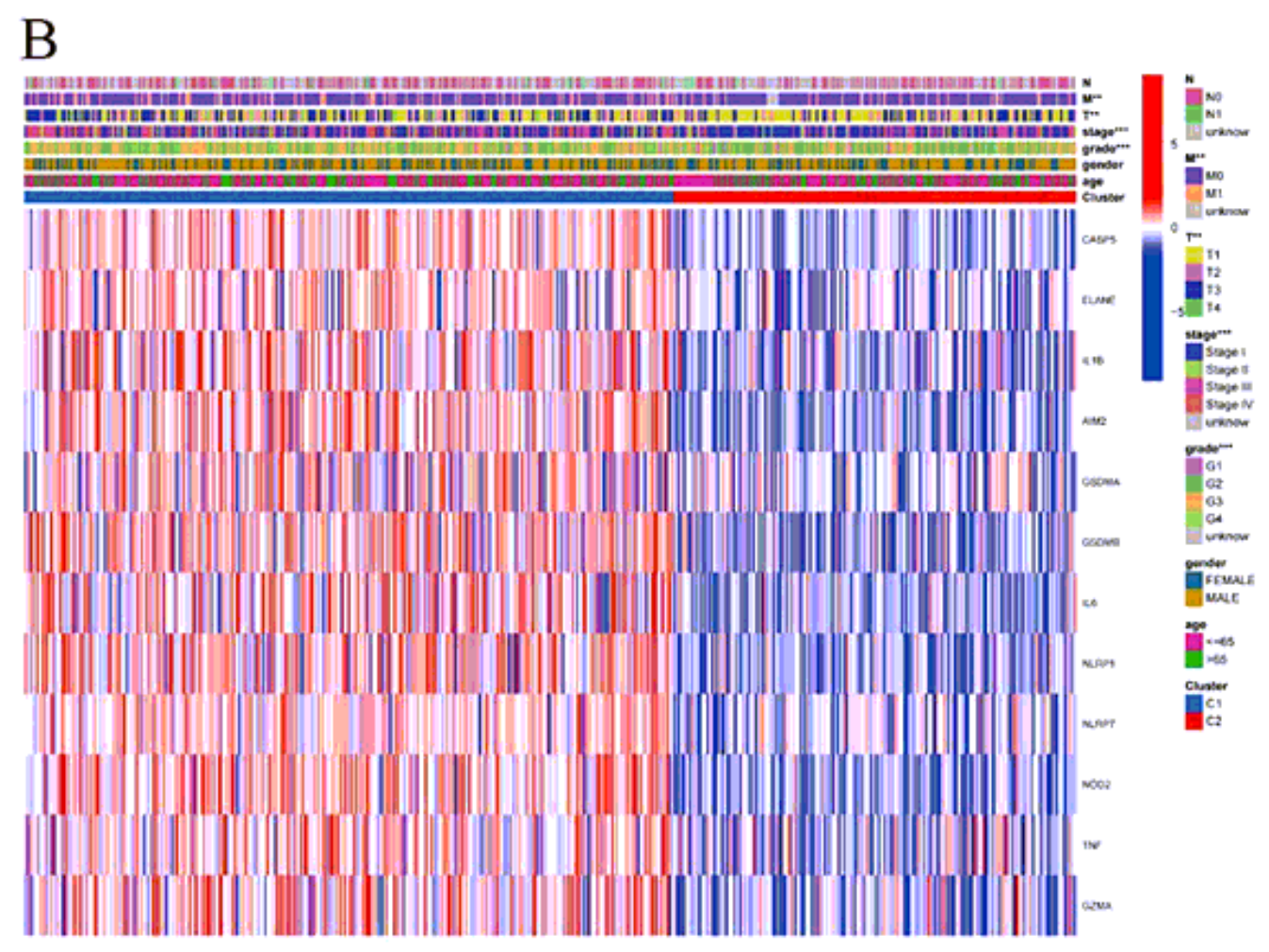

Figure 2

Tumor categorization according to the DEGs related to pyroptosis. A 539 ccRCC patients were assigned into 2 clusters based on the CCA matrix $(k=2)$. B The clinicopathologic features and heatmap of the 2 clusters categorized by DEGs ( $T, N$, and $M$ classification included lymph node metastasis, tumor size, and distant metastasis) (P values: ${ }^{*} P<0.05{ }^{*} \mathrm{P}<0.01$; ${ }^{\star \star *} \mathrm{P}<0.001$ ). C Kaplan-Meier OS curves for the 2 clusters. 
A

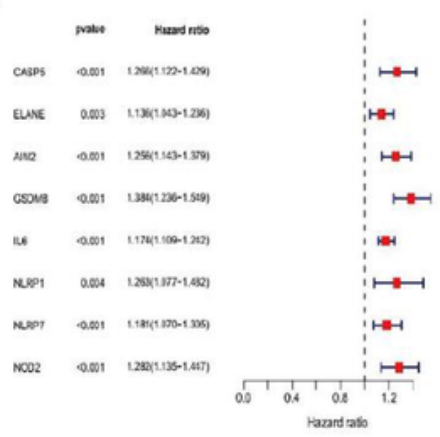

$\mathrm{C}$

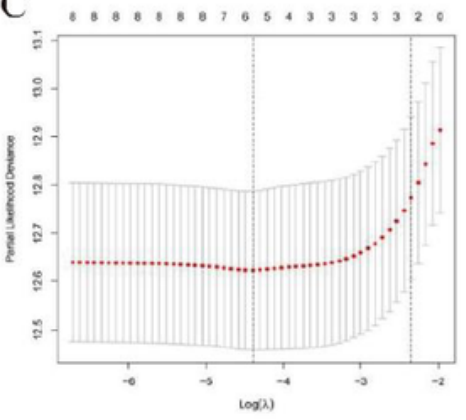

$\mathrm{E}$

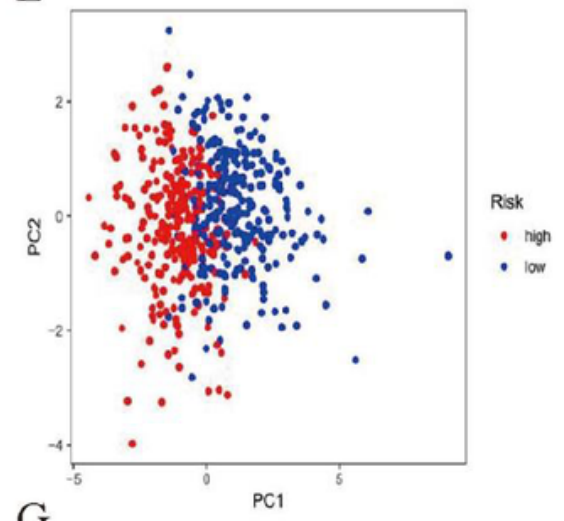

G

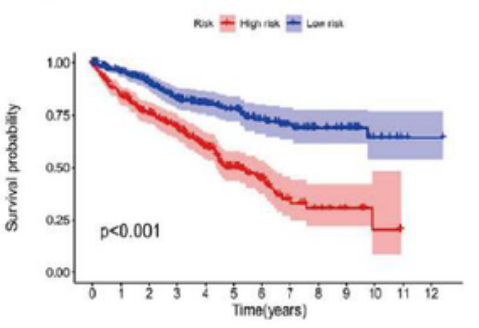

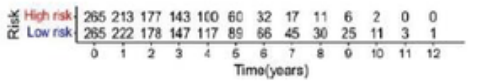

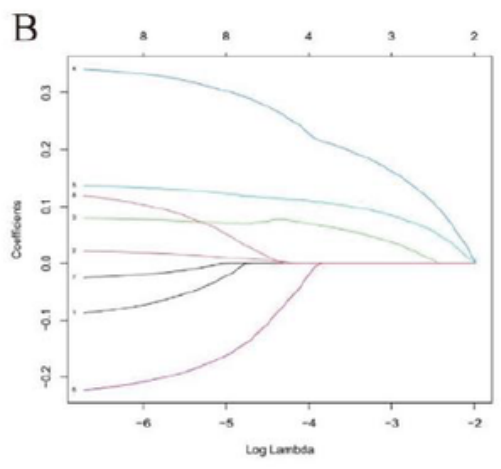

D

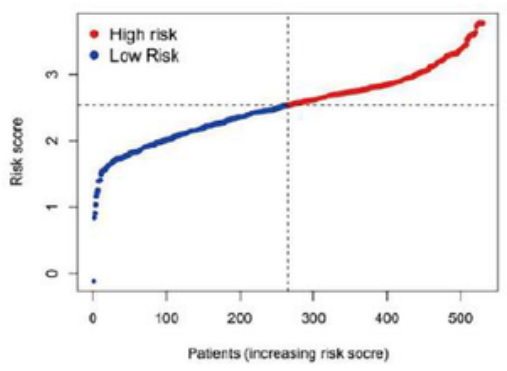

$\mathrm{F}$

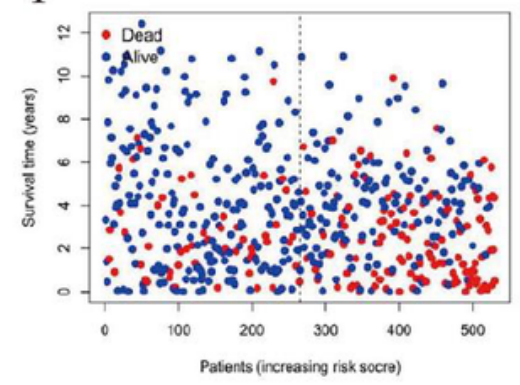

$\mathrm{H}$

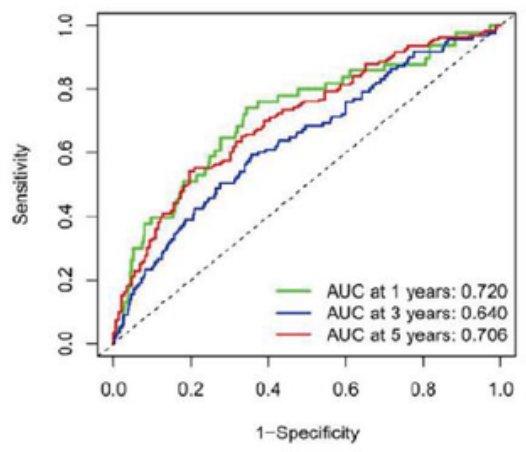

\section{Figure 3}

Risk signatures are constructed in the cohort of TCGA. A cCRCC analyzed by univariate cox regression for every DEG linked to pyroptosis, and 8 genes with P less than 0.05. B 6 genes linked to OS were analyzed by LASSO regression. C The selection of parameters was cross-validated. D Case distribution according to RS. E Principal component analysis for ccRCCs according to the RS. F Survival of every patient (the 
right side of the dark line, HR cases; the left side, LR cases). G KM curves for ccRCC cases in the LR group and HR group. H Predictive efficiency of the RS indicated by ROC curves.

A
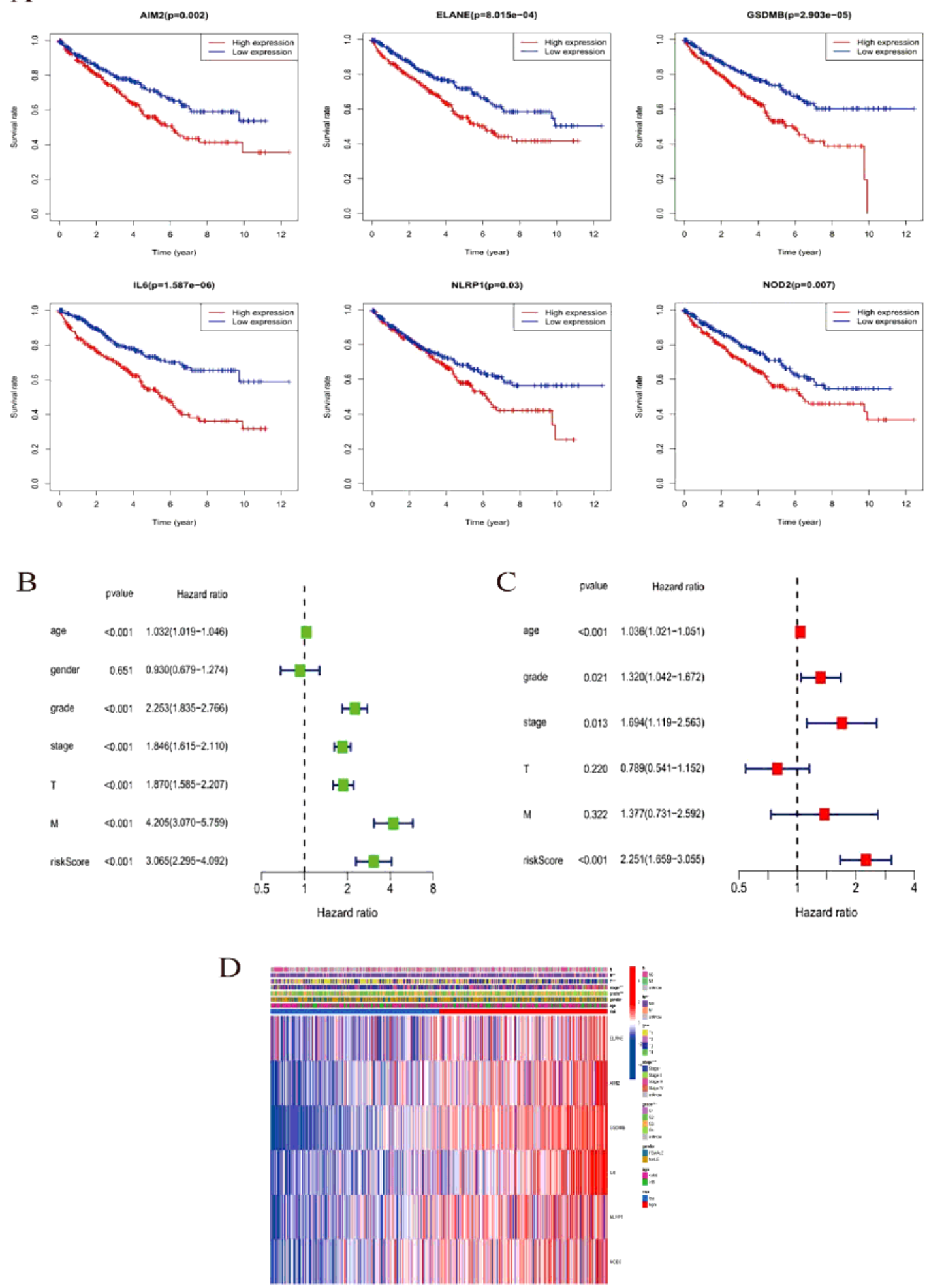

\section{Figure 4}

RS analyzed by Cox regression. A Kaplan-Meier analysis of the six hub genes linked to pyroptosis (ELANE, AIM2, GSDMB, IL6, NLRP1, and NOD2) in TCGA database. B Results of univariate analysis on the cohort of TCGA. C Results of multivariate analysis on the cohort of TCGA. D Heatmap (blue color, 
downregulated expression; red color, upregulated expression) for the relationships of clinical characteristics with the risk groups $(* \star * P<0.001, * * P<0.01, * P<0.05)$.

A

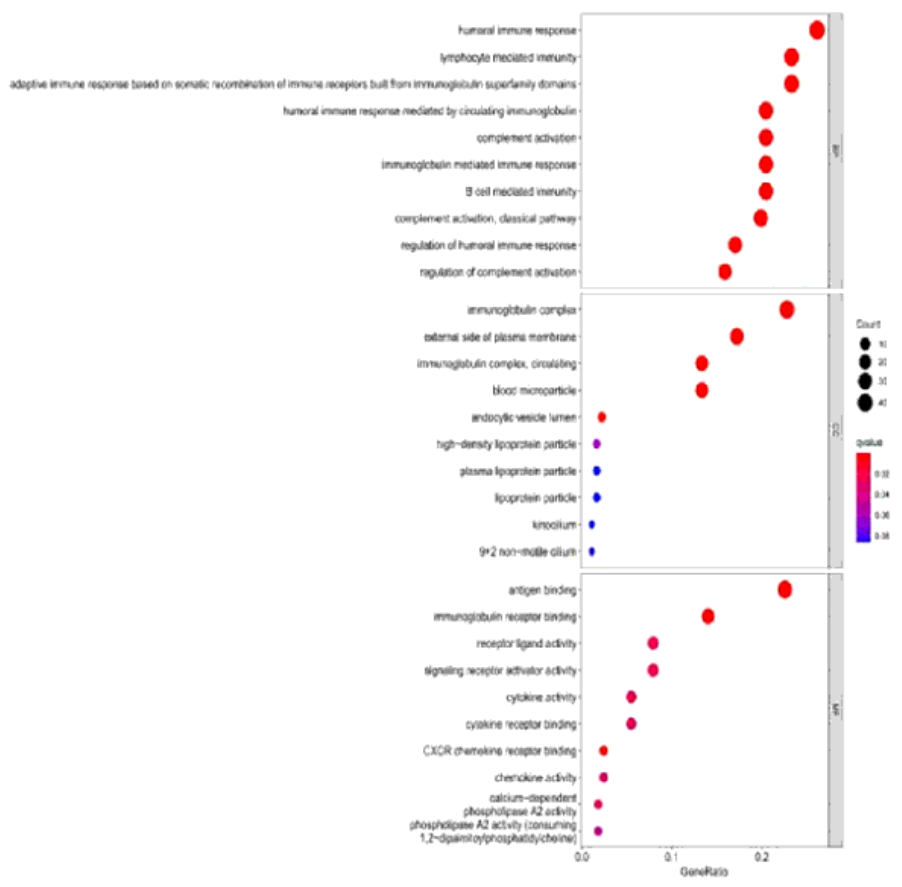

$\mathrm{B}$

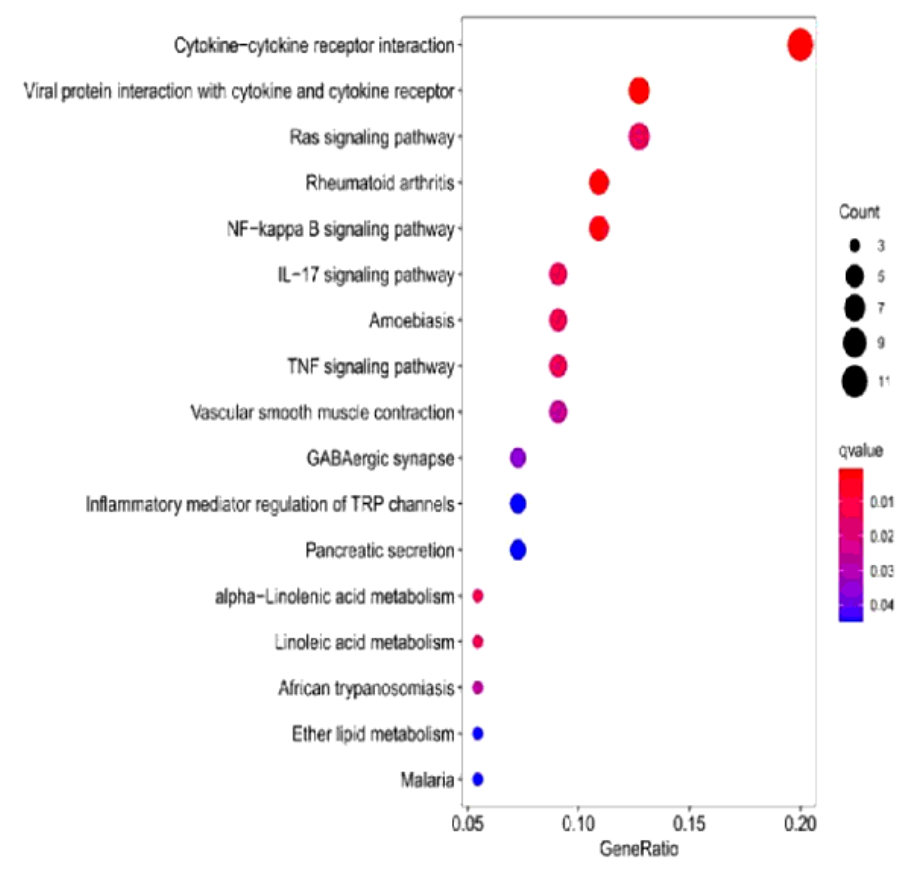

\section{Figure 5}

Analyses of functional enrichment. In details, A Bubble graph concerning GO enrichment. B Bubble graph concerning KEGG enrichment (more dark red indicates more noticeable differences, and bigger bubbles indicate more genes enriched; $\mathrm{MF}$, molecular function; $\mathrm{CC}$, cellular component; $\mathrm{BP}$, biological process; $\mathrm{q}$ value: the adjusted $p$-value). 
A

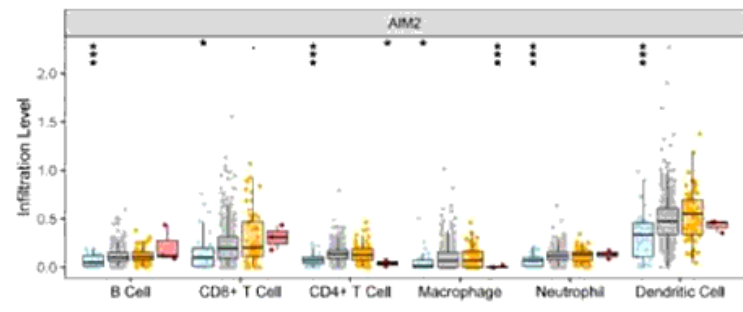

C

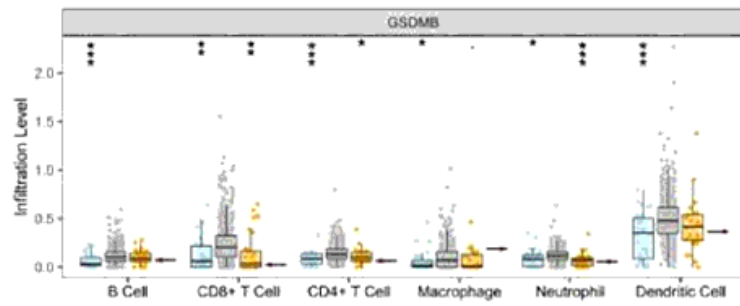

E

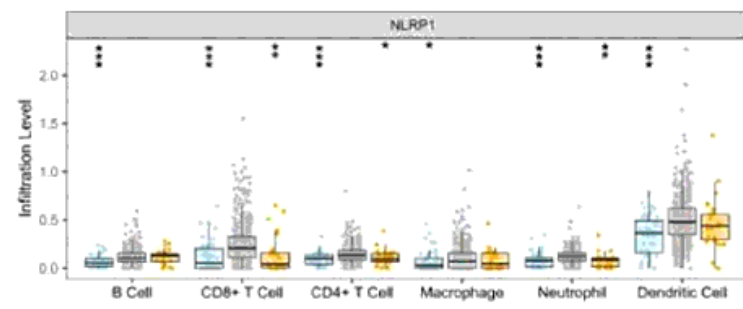

B

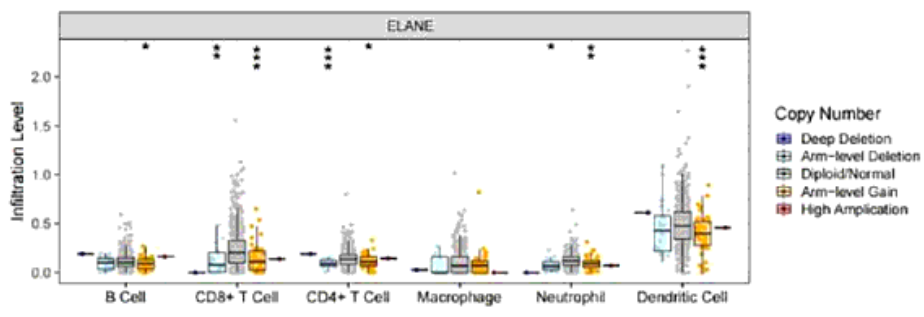

D

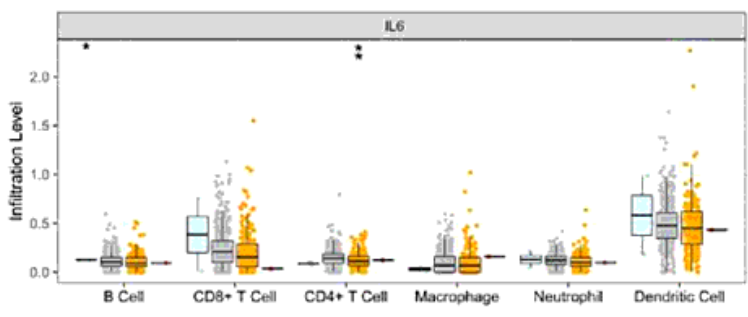

Copy Number 由 Arm-levol Deletion is Am-level Gain in High Amplication

F

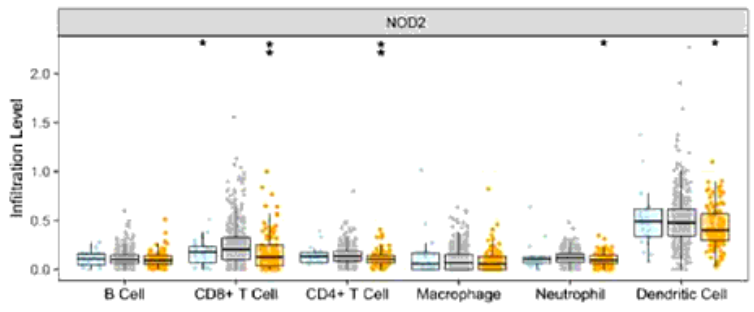

Copy Number if Arm-level Desetion 由.m-level Gain

\section{Figure 6}

Infiltration levels of immune cells of the six PRGs mutants linked to prognosis. A AIM2, B ELANE, C GSDMB, D IL6, E NLRP1, F NOD2. ***p $<0.001,{ }^{* *} \mathrm{p}<0.01,{ }^{*} \mathrm{p}<0.05$. 
A

Cor $=0.196(p=5.491 e-06)$

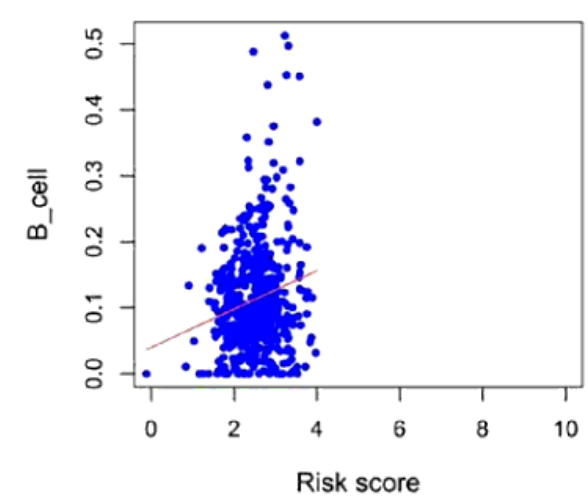

$\mathrm{D}$

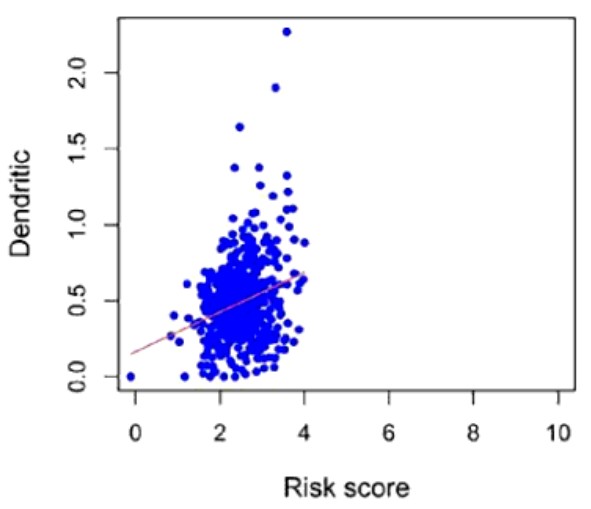

B

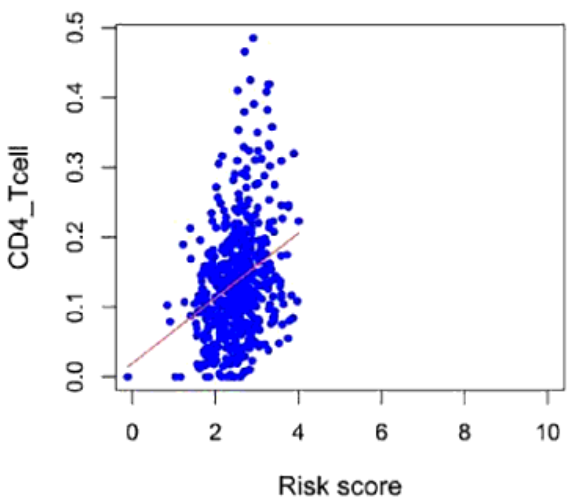

$\mathrm{E}$

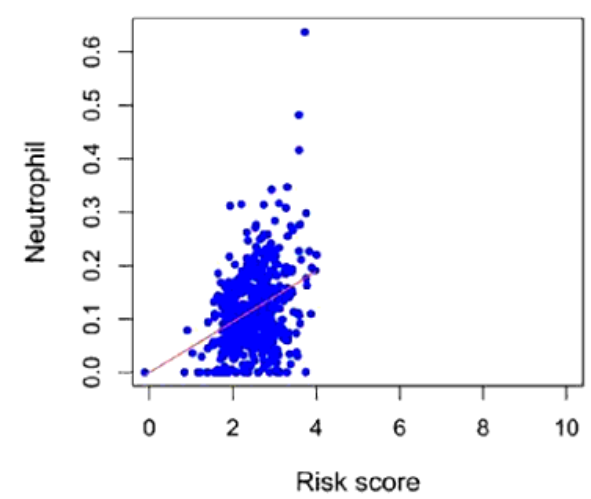

C Cor $=0.236(p=3.92 e-08)$

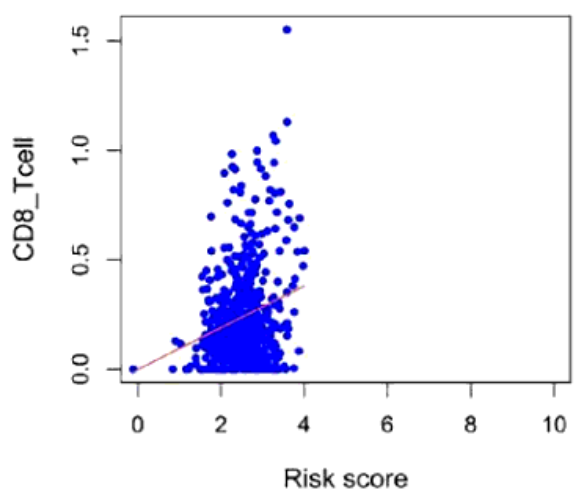

$\mathrm{F}$

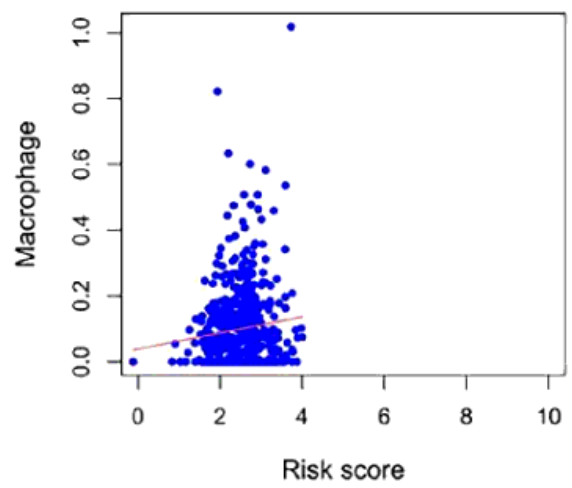

Figure 7

Correlation of risk scores with immune infiltration levels in ccRCCs. The risk score of the PRGs prognostic model were correlated positively with infiltration levels of immune cells (CD8+T cells, B cells, CD4+T cells, dendritic cells, neutrophils, and macrophages. Cor $>0, \mathrm{P}<0.05)$. 
A

Risk 它 low 帛 high

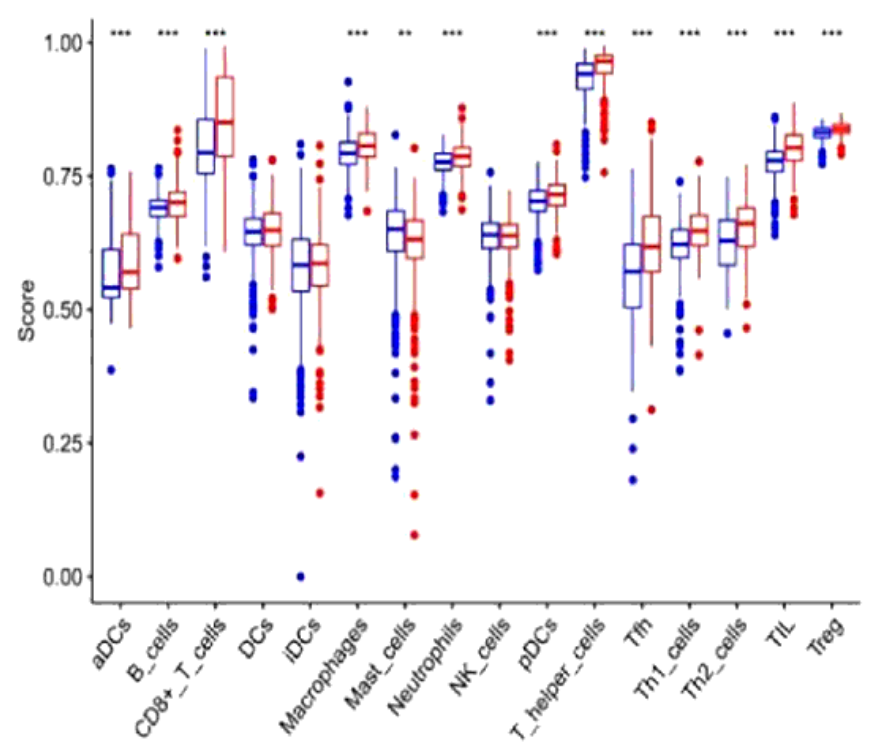

B

Risk 追 low 追 nigh

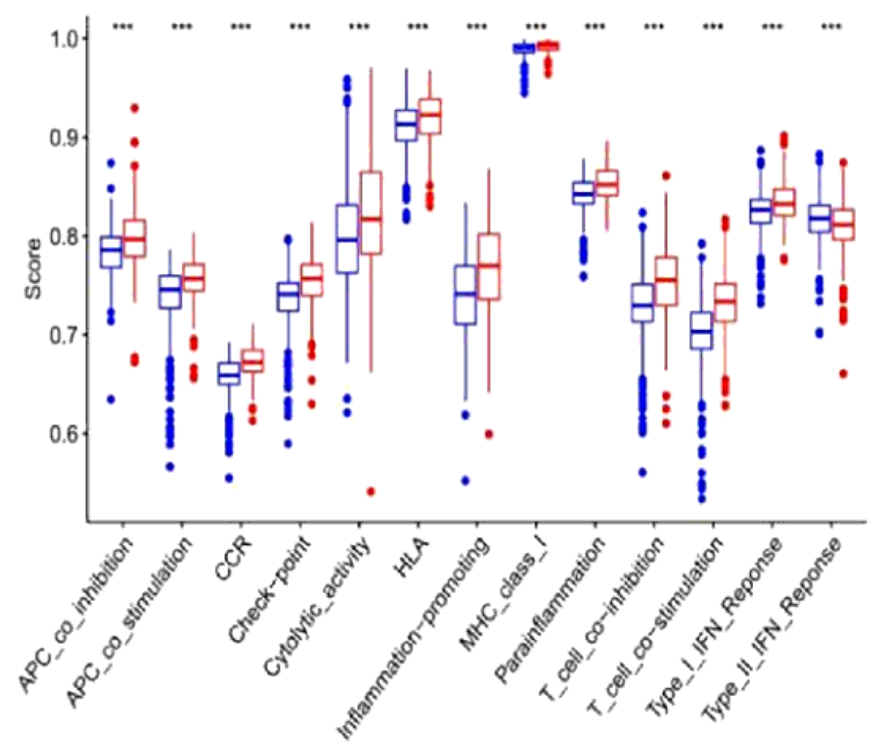

Figure 8

ssGSEA scores concerning immune pathways and cells. A Enrichment scores of sixteen types of immune cells between LR group (blue box) and HR group (red box). B Enrichment scores of thirteen pathways linked to immunity between high- (red box) and low- (blue box) risk groups. ${ }^{\star \star *} P<0.001,{ }^{\star *} P<0.01,{ }^{*} P<$ 0.05 . 


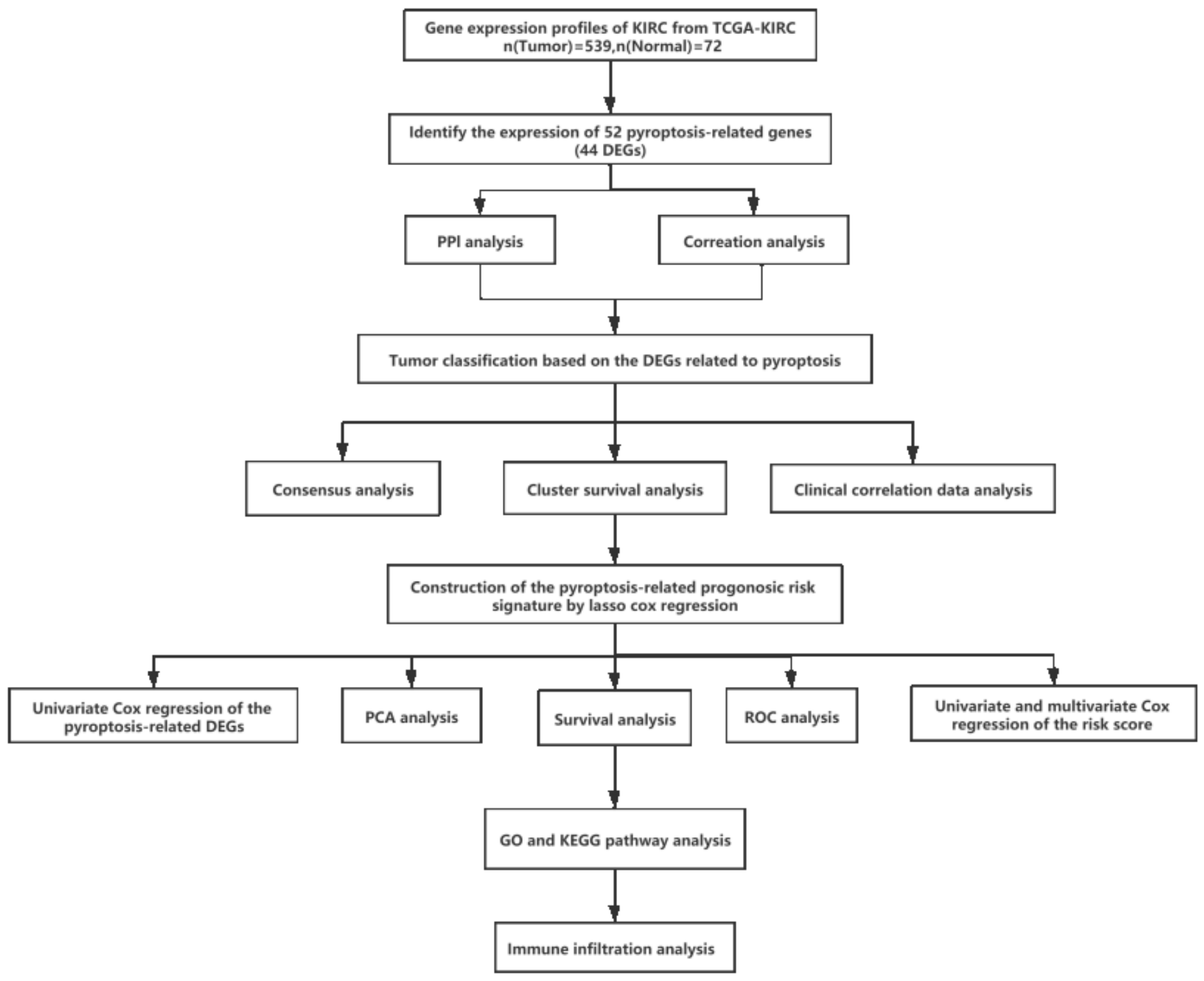

Figure 9

The specific data analysis workflow diagram.

\section{Supplementary Files}

This is a list of supplementary files associated with this preprint. Click to download.

- TableS152pyroptosisrelatedgenes.xlsx

- TableS2Markersofimmunecellsandpathways.xlsx

- TableS3DEGsbetweenhighandlowriskgroups.xlsx 